\title{
Calidad de vida pre y posadenoamigdalectomía en pacientes pediátricos
}

\section{Quality of life pre and post-adenotonsillectomy in pediatric patients}

\author{
Michel Royer $\mathrm{F}^{1}$, Héctor Bahamonde $\mathrm{S}^{1}$, Rossana Mamani $\mathrm{J}^{2}$, Rita Rodríguez $\mathrm{G}^{3}$, \\ Constanza Valdés $\mathrm{P}^{4}$, Consuelo Sanhueza $\mathrm{L}^{4}$.
}

\begin{abstract}
RESUMEN
La adenoamigdalectomía es la intervención quirúrgica más frecuente en otorrinolaringología, siendo su principal indicación las alteraciones obstructivas de la vía aérea superior asociadas con hiperplasia adenoamigdalina. 1 resultado posoperatorio habitualmente es evaluado de una forma subjetiva y no se ha estudiado sistemáticamente en nuestro país. En este estudio se utiliza una encuesta de calidad de vida validada en el extranjero (OSA-18), se realiza una prueba piloto y posteriormente se aplica en forma prospectiva a 64 tutores de pacientes pediátricos, previo a realización de adenoamigdalectomía y 3 meses tras la intervención. Adicionalmente se comparan los resultados con un grupo control de 63 niños asistentes a un jardín infantil $\theta$ puntaje promedio preoperatorio en la muestra fue de 4,3 (2,3-6,1), estableciendo diferencias significativas con el 2,47 (1,3-3,5) de los controles. 1 puntaje promedio de cambio con el posquirúrgico fue de 1,9. La encuesta demuestra un deterioro en alteraciones del sueño, síntomas físicos, emocionales y grado de preocupación del cuidador en niños con indicación quirúrgica por hiperplasia adenoamigdalina sintomática. Además la evaluación posoperatoria demuestra un beneficio significativo en diversos aspectos de la calidad de vida tras la intervención quirúrgica.

Palabras clave: Adenoamigdalectomía, Calidad de Vida.
\end{abstract}

\section{SUMMARY}

Adenotonsillectomy is the most frequent surgical operation in otolaryngology, its main indication being the obstructive alterations of the upper airway associated to adenotonsillar hyperplasia The post operatory result is normally evaluated in a subjective way and has not been systematically studied in our country. In this study, a

\footnotetext{
${ }^{1}$ Médico del Servicio de Otorrinolaringología. Hospital Cínico de la Universidad de Chile.

${ }^{2}$ Médico del Servicio de Pediatría. Hospital de Niños Dr. Roberto del Río

${ }^{3}$ Enfermera del Servicio de Otorrinolaringología. Hospital Cínico de la Universidad de Chile.

${ }^{4}$ Interna Escuela de Medicina. Facultad de Medicina Universidad de Chile
} 
survey on quality of life is utilized validated abroad (OSA-18), a pilot test is made and is later applied prospectively to 64 tutors of pediatric patients, before conducting the adenotonsillectomy and 3 months after the operation. Additionally the results are compared with a control group of 63 children from a nursery school. The average preoperatory score in the sample was 4,3 (2,3-6,1), establishing significant differences with the $2,47(1,3-3,5)$ of the controls. The average change score with the post surgical was 1,9. The survey shows a deterioration of sleep alterations, physical and emotional symptoms and degree of preoccupation of the children's ward with surgical indication due to symptomatic adenotonsillar hyperplasia Also the post-operatory evaluation shows a significant improvement in several aspects of the quality of life after the surgical operation.

Key words: Adenotonsillectomy, Quality of Life.

\section{INTRODUCCIÓN}

La adenoamigdalectomía es la intervención quirúrgica más frecuente en la práctica otorrinolaringológica, siendo sus indicaciones más aceptadas la infección crónica y la obstrucción de la vía aérea superior ${ }^{1}$. Esta última condición es de severidad variable, abarcando desde la roncopatía hasta el síndrome de apnea obstructiva del sueño, y se ha asociado con alteraciones en el comportamiento, disminución en el rendimiento escolar y deterioro en la calidad de vida ${ }^{2-6}$. La mejoría en diversos aspectos que abarca la calidad de vida es uno de los principales objetivos de la intervención quirúrgica en este tipo de pacientes.

Debe recordarse que la calidad de vida es un concepto que abarca los efectos funcionales de la enfermedad y del tratamiento en el paciente según su percepción. Esta subjetividad determina la necesidad de utilizar instrumentos estandarizados que permitan valorar de un modo más objetivo las percepciones del paciente y sus familiares. En cuanto al tratamiento, si el objetivo de éste es mejorar la calidad de vida, tal como se plantea con la adenoamigdalectomía, existe dificultad en decidir quiénes y en qué grado se benefician si no se cuenta con un elemento de medición adecuado.

Con el fin de determinar de una forma estandarizada el efecto de esta cirugía, se han construido diversos cuestionarios, siendo uno de los más utilizados el OSA-187 , de habla inglesa, validado para evaluar calidad de vida en pacientes pediátricos con apnea obstructiva del sueño, en cuanto a severidad ${ }^{7}$ y cambio longitudinal ${ }^{8}$. Este consta de 18 preguntas, divididas en cinco dominios (alteraciones en el sueño, síntomas físicos, emocionales, síntomas diurnos y grado de preocupación del cuidador), cada una con un puntaje de 1 a 7, correspondiendo el mayor valor a una condición más intensa.

Con ésta y otras herramientas se ha logrado demostrar una mejoría en la calidad de vida posoperatoria en población infantil sometida a adenoamigdalectomía por causas obstructivas ${ }^{6,9-}$ 13, como también en adultos por amigdalitis a repetición ${ }^{14}$. Sin embargo, también existen reportes que demuestran que el cambio sustancial es a corto plazo y no se relaciona a todos los aspectos controlados, como alteraciones emocionales y la preocupación de los cuidadores ${ }^{15}$.

En nuestro país, si bien la adenoamigdalectomía es la intervención otorrinolaringológica más frecuente $^{16}$ no existe información acerca del efecto de la cirugía sobre la calidad de vida, por lo que parece necesario conocer el efecto sobre nuestros pacientes y sobre qué aspectos realmente estamos actuando. Esta investigación buscará traducir el cuestionario OSA-18, verificar la aplicabilidad en nuestra población, para posteriormente realizar una comparación de calidad de vida pre y posadeno- 
amigdalectomía en un grupo de pacientes pediá tricos con hiperplasia adenoamigdalina.

\section{MATERIAL Y MÉTODO}

Se diseña un estudio prospectivo, a realizar durante los meses de marzo a septiembre de 2005. En marzo se obtiene el cuestionario OSA-18, se realiza su traducción al idioma español y se aplica en 30 tutores de pacientes pediátricos sometidos a adenoamigdalectomía mediante una prueba piloto, en la cual se verifican las dificultades en responderla, términos poco comprensibles, tiempo requerido y necesidad de ayuda.

Durante los meses de abril a junio de 2005 se ingresan al estudio pacientes menores de 12 años con indicación de adenoamigdalectomía por hiperplasia adenoamigdalina y síntomas obstructivos de la vía aérea superior. A éstos se les realiza una evaluación otorrinolaringológica, se completa un formulario individual con sus características clínicas (hallazgos al examen físico, tamaño amigdalino, patologías coexistentes y antecedentes generales) y estudios realizados (radiografía de cavum, nasofibroscopía). Sus tutores responden previo a la intervención quirúrgica una encuesta de calidad de vida (OSA-18), en forma individual y autoaplicada Posteriormente la misma persona se encuestará telefónicamente a los 3 meses tras la intervención, en los meses de julio a septiembre de 2005.

Se define un grupo control, con niños asistentes al Jardín Infantil del Hospital Cínico de la Universidad de Chile, cuyos tutores contestaron la encuesta (OSA-18).

Se establecen como criterios de exclusión de ambos grupos: cirugía adenoidea/amigdalina previa, alteraciones craneofaciales, enfermedades neuromusculares, fisura palatina o cirugía velofaríngea previa, déficit cognitivo conocido, enfermedad psiquiátrica e incapacidad de comprensión del idioma por parte de cuidadores.

Para el análisis de severidad se considera el puntaje total de la encuesta, clasificando el impacto en la calidad de vida en leve (menor de 60), moderado (entre 60 y 80 ) y severo (sobre 80$)^{7}$.
Los puntajes obtenidos se compararon mediante z de promedios, considerándose significativo un $p$ $<0,05$. En el seguimiento longitudinal (pre y posquirúrgico) se utilizan los puntajes promedio por pregunta, y en una escala de 1 a 7 , se considera que la diferencia es insignificante si es menor de 0,5 , leve $(0,5-1,0)$, moderada $(1,0-1,5)$ o grande (mayor de 1,5$)^{8}$.

\section{RESULTADOS}

Inicialmente se obtuvo el cuestionario OSA-18, se tradujo al idioma español (Tabla 1) y se realizó una prueba piloto con 30 tutores de pacientes pediátricos sometidos a adenoamigdalectomía, la que demostró aplicabilidad de la encuesta en nuestra población. Estos tutores respondieron la encuesta de forma personal, sin requerir ayuda externa, en menos de cinco minutos y refirieron escasas dificultades, ninguna de las cuales impidió contestarla de forma adecuada. Se realizaron pe queños cambios en términos médicos, los que en suma no alteraron la encuesta, siendo concordante esta versión en español con la original en inglés.

Durante el período abril a junio de 2005 se encuestaron a 64 tutores de niños sometidos a adenoamigdalectomía por hiperplasia adenoamigdalina y síntomas obstructivos de la vía aérea superior. También se aplicó el cuestionario a 63 tutores del grupo control, constituido por asistentes al Jardín infantil del Hospital Cínico de la Universidad de Chile.

La muestra de 64 pacientes presentaba una edad promedio de 5 años (rango 2-10 años), sin diferencias entre hombres y mujeres. 1 grupo control era comparable en distribución por sexo y edad.

Entre los antecedentes relevantes de la muestra de pacientes el $23 \%$ presentaba antecedentes o cuadro concurrente otológico (otitis media aguda a repetición u otitis media con efusión), $17 \%$ era portador de rinitis y $7 \%$ refería cuadros bronquiales obstructivos a repetición. En el examen de cavidad oral el $41 \%$ presentaba amígdalas grado III y $39 \%$ grado IV. 
Tabla 1. Encuesta Calidad de Vida (OSA - 18)

Encuesta Calidad de Vida (OSA -18)

ANEXO1

Nombre Paciente:

RUT Paciente:

Para cada una de las preguntas siguientes, marque el número de veces que mejor describe cuán frecuente cada síntoma o problema le ha ocurrido en las últimas cuatro semanas. Por favor, marque sólo un número por pregunta. Gracias.
Considere: $\quad$ 1.- Nunca
2.- Muy alejado
3.- Pocas veces
4.- Algunas veces
5.- A menudo
6.- La mayoría
7.- Siempre

\section{Síntomas del Paciente}

Durante las 4 últimas semanas cuán frecuente su hijo ha presentado:

Ronquidos fuertes?

Períodos de apnea o pausas respiratorias en la noche?

Ahogos o sonidos de jadeo mientras duerme?

Sueño no restaurador o frecuentes despertares nocturnos?

$\begin{array}{lllllll}1 & 2 & 3 & 4 & 5 & 6 & 7 \\ 1 & 2 & 3 & 4 & 5 & 6 & 7 \\ 1 & 2 & 3 & 4 & 5 & 6 & 7 \\ 1 & 2 & 3 & 4 & 5 & 6 & 7\end{array}$

Durante las 4 últimas semanas cuán frecuente su hijo ha presentado:

Respiración bucal debido a obstrucción nasal?

Resfríos frecuentes o infecciones respiratorias altas?

Nariz congestionada o rinorrea (secreción nasal)?

Dificultad para tragar la comida?

$\begin{array}{lllllll}1 & 2 & 3 & 4 & 5 & 6 & 7 \\ 1 & 2 & 3 & 4 & 5 & 6 & 7 \\ 1 & 2 & 3 & 4 & 5 & 6 & 7 \\ 1 & 2 & 3 & 4 & 5 & 6 & 7\end{array}$

Durante las 4 últimas semanas cuán frecuente su hijo ha presentado:

Cambios de ánimo o pataletas/rabietas?

Comportamiento agresivo o hiperactivo?

Problemas disciplinarios?

$\begin{array}{lllllll}1 & 2 & 3 & 4 & 5 & 6 & 7 \\ 1 & 2 & 3 & 4 & 5 & 6 & 7 \\ 1 & 2 & 3 & 4 & 5 & 6 & 7\end{array}$

Durante las 4 últimas semanas cuán frecuente su hijo ha presentado:

Excesivo sueño durante el día?

Dificultad en la concentración?

Dificultad para levantarse en las mañanas?

$\begin{array}{lllllll}1 & 2 & 3 & 4 & 5 & 6 & 7 \\ 1 & 2 & 3 & 4 & 5 & 6 & 7 \\ 1 & 2 & 3 & 4 & 5 & 6 & 7\end{array}$

Preocupaciones del familiar

Durante las 4 últimas semanas cuán frecuente a usted se le han presentado los siguientes problemas:

Preocupación por el estado general de salud de su hijo?

Le ha preocupado que su hijo no reciba suficiente aire?

Le ha interferido con la realización de sus actividades diarias?

$\begin{array}{lllllll}1 & 2 & 3 & 4 & 5 & 6 & 7\end{array}$

Le ha causado frustración?

$\begin{array}{lllllll}1 & 2 & 3 & 4 & 5 & 6 & 7\end{array}$

$\begin{array}{lllllll}1 & 2 & 3 & 4 & 5 & 6 & 7\end{array}$

Nombre Encuestado

Parentesco

Teléfono

E-Mail 
日 $80 \%$ de la muestra de pacientes presentaba un puntaje total mayor de 60 , por lo que se distribuían en un impacto moderado o severo sobre su calidad de vida. $\mathrm{\theta}$ puntaje total promedio prequirúrgico fue de 74 . En cambio, en el grupo control, el $80 \%$ estaba bajo 60 en el puntaje total, por lo que se encontraban en un impacto leve (Figura 1) y su puntaje total promedio como grupo era de 43 , diferencia estadísticamente significativa con relación al grupo de pacientes.

Al analizar los puntajes por pregunta en los diferentes dominios: alteraciones en el sueño, físicas, emocionales, síntomas diurnos y preocupa- ción del cuidador, los promedios más altos se encuentran en síntomas físicos y preocupación del cuidador (para el grupo de pacientes) y en preocupación del cuidador y síntomas emocionales (en los controles). En todos los dominios, salvo en síntomas diurnos, existen diferencias significativas entre los grupos (Fgura 2).

Para determinar el cambio posquirúrgico se utilizaron los puntajes promedios por pregunta. En el grupo de pacientes el puntaje prequirúrgico fue de 4,03 y el posquirúrgico de 2,13; estableciendo una diferencia de 1,9 considerado un gran cambio en calidad de vida según la escala de seguimiento

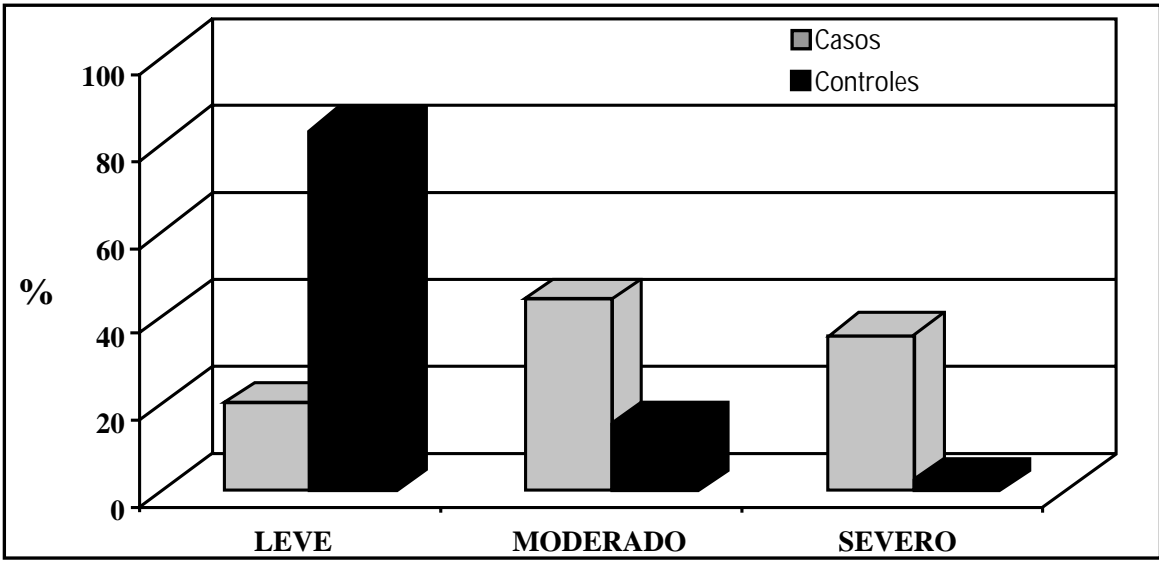

Figura 1. Impacto en calidad de vida

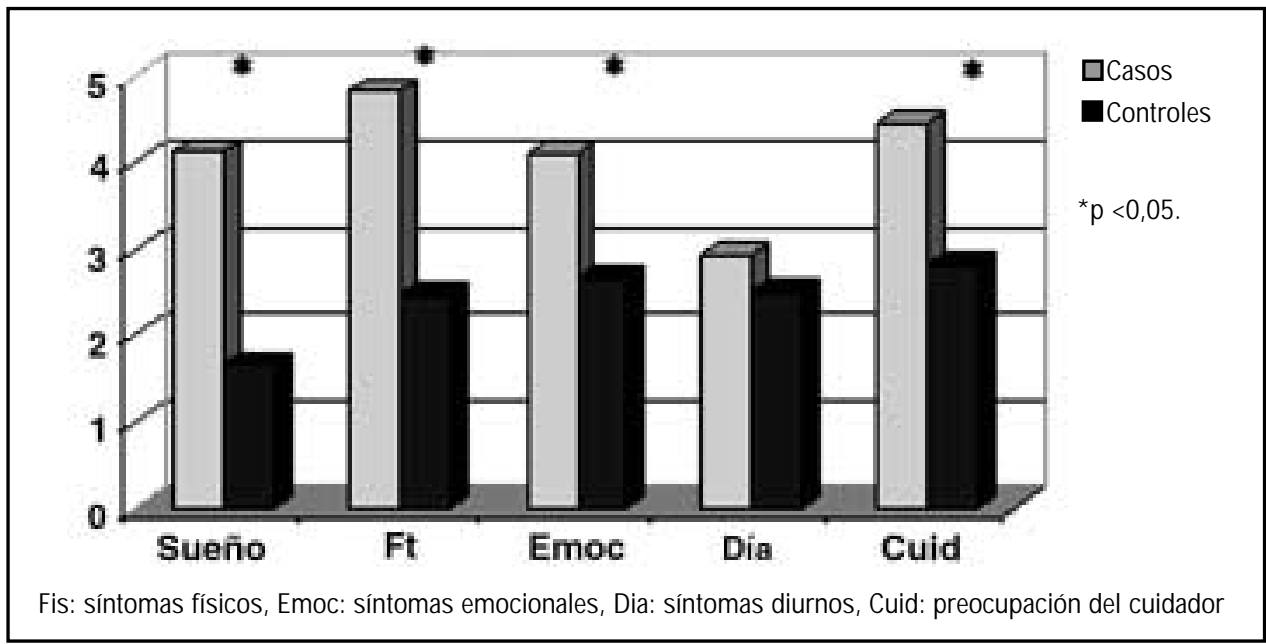

Figura 2. Puntaje promedio por dominios 
longitudinal (Figura 3). Además se debe destacar que este puntaje postoperatorio es similar al 2,47 obtenido por el grupo control.

A analizar los diferentes dominios del cuestionario se obtienen diferencias estadísticamente significativas prey posintervención en todos los estudiados, salvo en síntomas diurnos (Figura 4). Consistentemente, los valores obtenidos posoperatorios son similares a los del grupo control.

Si se considera el puntaje total tres meses posintervención quirúrgica en el grupo de pacientes para valorar la severidad sobre la calidad de vida, el $88 \%$ del grupo se encuentra en un impacto leve, $12 \%$ en moderado y $0 \%$ en severo. 日 puntaje total promedio a tres meses de la adenoamigdalectomía es de 38, siendo significativa su diferencia con el preoperatorio y sin diferencias con el grupo control

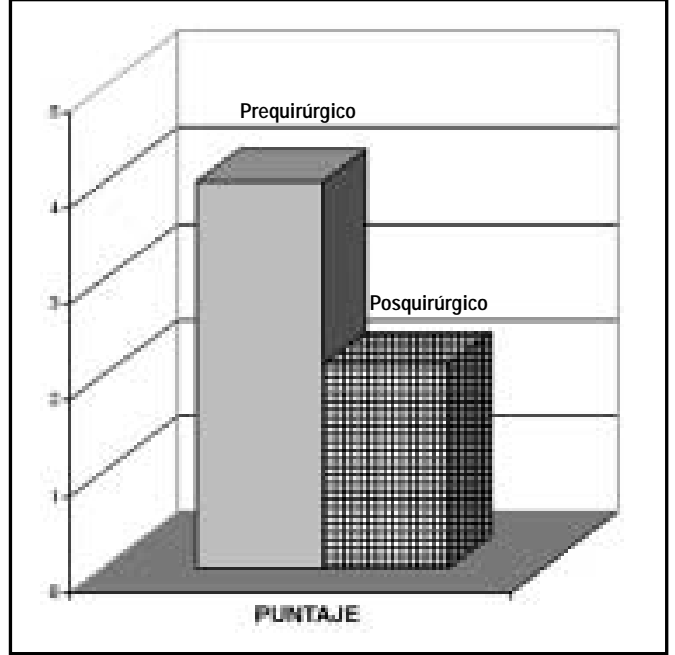

Figura 3. Puntaje promedio por pregunta.

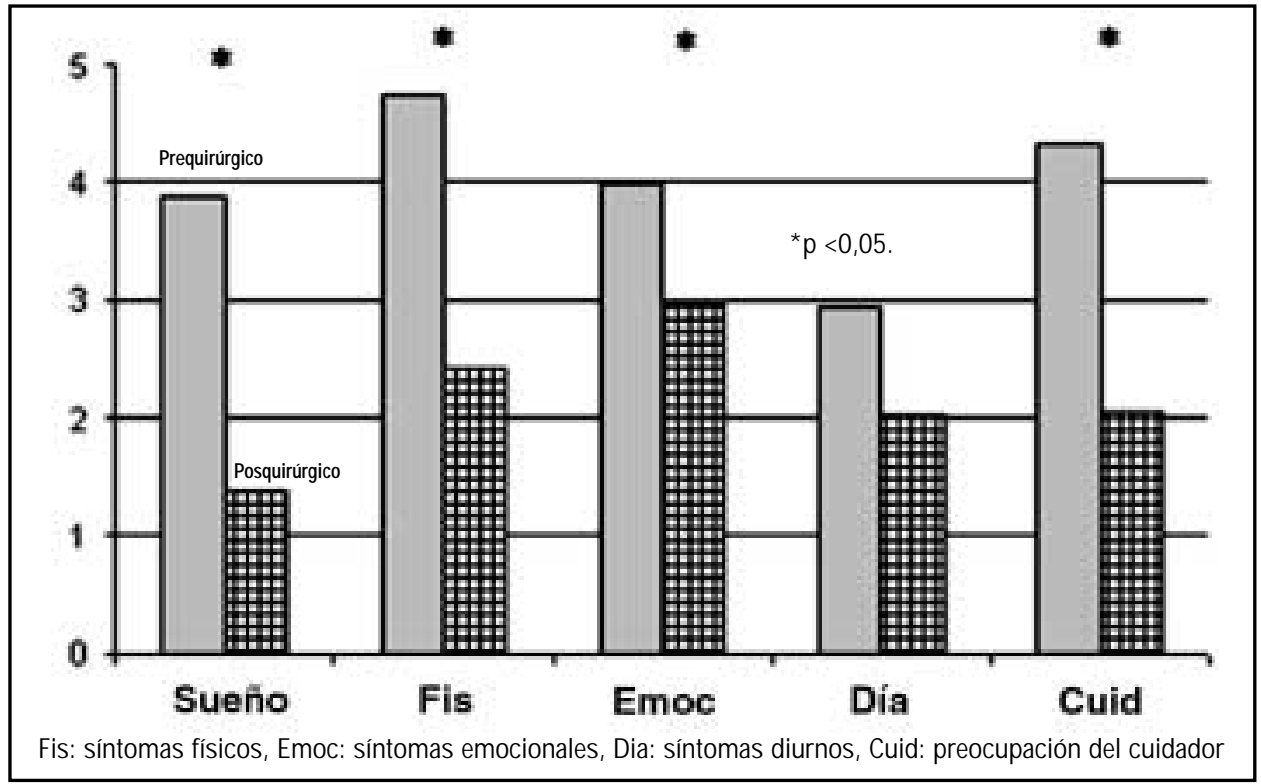

Figura 4. Puntaje promedio por dominios.

\section{DISCUSIÓN}

La adenoamigdalectomía es la intervención quirúrgica más frecuente en otorrinolaringología, siendo su principal indicación la hiperplasia adenoamigdalina con alteraciones obstructivas durante el sueño, las que se asocian con un deterioro en la calidad de vida de los pacientes, alteraciones en el comportamiento y deterioro del rendimiento esco$\operatorname{lar}^{2-6}$.

日 objetivo de mejorar la calidad de vida en estos pacientes, tradicionalmente se evalúa en 
forma subjetiva en los controles posoperatorios, por lo que se puede ver influida por múltiples factores, tales como cercanía temporal al evento, dificultad para describir situación previa, complicaciones y relación médico-paciente. Dado este contexto, cuantificar el cambio de forma más objetiva y realista requiere del uso de un elemento que se aplique en un tiempo previo y luego posintervención. En este sentido el cuestionario OSA 18 parece una herramienta apropiada ya que abarca diferentes aspectos de la vida de los pacientes pediátricos y se correlaciona con hallazgos al examen físico, nasofibroscopía y polisomnografía, encontrándose validado tanto para evaluar severidad como seguimiento longitudinal ${ }^{7,8}$.

No existen experiencias nacionales previas con instrumentos estandarizados, por lo que nos pare ce apropiado adaptar el OSA 18 a nuestra realidad, dado que esto nos permite tener una herramienta ya establecida y que nuestros resultados sean comparables a experiencias de otros países. $\mathrm{Si}$ bien esta encuesta está diseñada en niños con síndrome de apnea obstructiva del sueño, la aplicación de ella en patología obstructiva del sueño, en general, parece adecuada dado que, en la práctica, no se solicitan polisomnografías para indicar la cirugía en la gran mayoría de estos pacientes.

La prueba piloto realizada como experiencia inicial en este proyecto demostró la aplicabilidad de esta encuesta en nuestra población, ya que fue contestada en corto tiempo, en forma personal y sin mayores dificultades por los tutores de los pacientes. Este punto es relevante, ya que nos permite disponer para nuestra práctica de esta herramienta como una forma de acercarnos a los verdaderos efectos de la intervención sobre nuestros pacientes. Debe considerarse que la población que atiende nuestro centro hospitalario corresponde en su mayoría a clase media chilena, y en los antecedentes aportados por los tutores se desprende que el $20 \%$ de ellos tenía educación básica, $40 \%$ educación media y $40 \%$ superior.

La aplicación de esta encuesta en la muestra estudiada demostró una peor calidad de vida de los pacientes pediátricos con alteraciones obstructivas de la vía aérea superior en comparación a un grupo control en múltiples aspectos evaluados: síntomas nocturnos, físicos, emocionales y preocupación del cuidador. $日$ único tópico en que no hubo diferencias significativas fue en la presencia de síntomas diurnos. Esto puede explicarse dado que las alteraciones del sueño en niños, sean roncopatías o síndrome de apnea obstructiva, no se asocian significativamente a somnolencia diurna, difiriendo de lo observado clásicamente en adultos $^{17}$. Debe destacarse que en el grupo control utilizado no se excluyeron niños, salvo según los criterios enunciados previamente, dado que nos pareció interesante que se asemejara a la población general, por lo que es esperable que en ellos exista un porcentaje de hiperplasia adenoamigdalina sintomática.

La aplicación de la encuesta en forma posoperatoria demostró una mejoría significativa en los aspectos estudiados, constatándose la similitud entre el grupo de pacientes posoperados y el grupo control, lo que apoya los efectos beneficiosos de la adenoamigdalectomía en este grupo de pacientes. En este sentido apoyamos la realización de adenoamigdalectomía en pacientes con hiperplasia adenoamigdalina y síntomas obstructivos de la vía aérea superior, con la evidencia necesaria de una mejoría en la calidad de vida, tanto del paciente como en aspectos relacionados al cuidador.

Entre las limitaciones del estudio debemos considerar que la aplicación de la encuesta tres meses poscirugía es un período cercano a la intervención terapéutica y la mejoría en la calidad de vida pudiera verse relacionada a otros factores, así como no se puede precisar si este cambio será estable en el tiempo. Además, siendo la patología adenoamigdalina la más frecuente de la especialidad, el tamaño muestral si bien es comparable a series internacionales y logra determinar diferencias estadísticas, es pequeño con relación a su prevalencia en la población.

Nos parece sumamente interesante el tener una herramienta que permita objetivar la severidad y el cambio longitudinal en un grupo de pacientes que será sometido a una intervención quirúrgica. Este punto abre variadas posibilidades para desarrollar 
investigaciones futuras, utilizar otro grupo de pacientes, valorar la severidad, identificar fracasos quirúrgicos y evaluar el cambio en los diferentes aspectos a tiempos de seguimiento mayores.

\section{BIBLIOGRAFÍA}

1. MatTlLa PS, TAHKOKALlio O, TARKKANEN J, PItANieMI J, KaRVONen M, TuOMILerTO J. Causes of tonsillar disease and frequency of tonsillectomy operations. Arch Otolaryngol Head Neck Surg 2001; 127 (1): 37-44.

2. ALI NJ, PITSON DJ, StRADLING JR. Snoring, sleep disturbance, and behaviour in 4-5 year olds. Arch Dis Child 1993; 68 (3): 360-6.

3. GR⿴囗十⿰ MG CARROLL JL. Consequences of sleep disordered breathing in childhood. Ourr Opin Pulm Med 1997; 3 (6): 456-63.

4. GOZAL D. Sleep-disorder breathing and school performance in children. Pediatrics 1998; 102: 616-20.

5. RHODES SK, SHMODA KC, WAID LRET AL. Neurocognitive deficits in morbidly obese children with obstructive sleep apnea. $J$ Pediatr 1995; 127 (5): 741-4.

6. Goldsten NA, Fatima M, Campbel L TF, Rosen田D RM. Child behavior and quality of life before and after tonsillectomy and adenoidectomy. Arch Otolaryngol Head Neck Surg 2002; 128 (7): 770-5.

7. Franco RA JR, ROSENFID RM, RaO M. Frst place-resident clinical science award 1999. Quality of life for children with obstructive sleep apnea. Otolaryngol Head Neck Surg 2000; 123 (1): 9-16

8. SOHN H, ROPSENRte R. Evaluation of sleep disordered breathing in children. Aolaryngology Head Neck Surg 128 (3): 344-52
9. Goldsten NA, Post JC, Rosenfled RM, CamPBEL TF. Impact of tonsillectomy and adenoidectomy on child behavior. Arch Otolaryngol Head Neck Surg 2000; 126 (4): 494-8.

10. Tran KD, NGuYen CD, WEDON J, GOLdSTEn NA. Child behavior and quality of life in pediatric obstructive sleep apnea. Arch Oolaryngol Head Neck Surg 2005; 131(1): 52-7.

11. MITCHEL RB, Ke⿴lY J, CALL E, YAO N. Quality of life after adenotonsillectomy for obstructive sleep apnea in children. Arch Otolaryngol Head Neck Surg 2004; 130 (2): 190-4.

12. Stewart M, Gaze D, Friedman E ÓBrien E BAUTISTA M. Quality of Life and Sleep Study findings after adenotonsillectomyin children with obstructive sleep apnea. Arch Aolaryngol Head Neck Surg 2005; 131: 308-14.

13. MITCHEL RB, Ke⿴lY J. Outcome of adenotonsillectomy for severe obstructive sleep apnea in children. Int $J$ Pediatrics Aorhinolaringology 2004; 68: 1375-79.

14. BHATTACHARYYA N, KENES LJ, SHAPIRO J. Efficacy and quality-of-life impact of adult tonsillectomy. Arch Otolaryngol Head Neck Surg 2001; 127 (11): 1347-50.

15. MITCHEL RB, KELY J, CALL E, YAO N. Longterm changes in quality of life after surgery for pediatric obstructive sleep apnea. Arch Otolaryngol Head Neck Surg 2004; 130 (4): 409-12.

16. Ulloa P, UrRa A, Valenzuea A, Benavente J. Análisis de la atención intrahospitalaria del Servicio de Otorrinolaringología, Hospital Guillermo Grant Benavente de Concepción, 19931997. Rev Atorrinolaringología Cir Cabeza Quello 1999; 53: 93-100.

17. GOZAL D, WANG M, POPE DW JR. Objective sleepiness measures in pediatric obstructive sleep apnea. Pediatrics 2001; 108: 693-7. 